\title{
The Secondary Stars in Short Period (Magnetic) Cataclysmic Variables
}

\author{
Steve B. Howell \\ University of California, Riverside, CA 92521
}

\begin{abstract}
We review the recent knowledge base pertaining to causes for mass accretion variations (high/low states) in highly magnetic cataclysmic variables (i.e. polars). We then examine what theory has to say about the properties of the secondary stars (the mass donors) with respect to those CVs with very short orbital periods. Finally, we use recent observations of EF Eri in an extended low state to provide direct observational evidence which shows that this $\mathrm{CV}$, as well as a few others, likely contain very low mass, sub-stellar secondary stars.
\end{abstract}

\section{Introduction}

Mass accretion in cataclysmic variables (CVs) has been extensively studied in terms of their accretion disk and the theorized relation between mass transfer rate and orbital period. Of course, the mass that is transfered comes from the companion star and its rate, as well as its character, are highly related to its evolutionary status. In the past few years, studies aimed at direct observation of the secondaries in CVs have been undertaken in an attempt to confirm theoretical predictions for these stars.

For the short period CVs, the topic under consideration herein, there are few cases of direct observation of their secondary, limited mostly to brief glimpses during eclipse or serendipitous observation during low states in magnetic systems. Purposeful observational programs which use target of opportunity time to observe magnetic CVs during low state have been started and they offer great hope in terms of providing detailed spectroscopic views of the mass donors.

Low states, times of reduced mass transfer from the secondary star, are an accepted observational property of CVs which contain white dwarfs harboring a high magnetic field. The systems we speak of are called polars or AM Herculis binaries and the white dwarf fields are 10-200 MG in strength. It is probably the case that intermediate polars and non-magnetic CVs have low states too, but they would be unobservable to the casual observer due to the presence of the accretion disk (King \& Cannizzo 1998).

We briefly examine below, the present day ideas as to a cause for these low states, actually for the entire suite of high, low, and in between mass transfer states. We then look at what theory has to say about the mass donors in both the pre- and post-orbital period minimum systems. Finally, we give some specific details about the polar EF Eri, lately sky-rocketed into stardom due 
to its seven year low state and powerful observational evidence for a brown dwarf-like secondary star.

\section{What is the Cause for High/Low State Behavior?}

Starting with initial works such as those of Bianchini (1992) and Hessman et al. (2000), evidence began to emerge that seemed to imply a possible relation between mass accretion variations (in particular low states) and solar cycle type phenomena. The evidence showed variations on the timescales of decades in overall system brightness and gave theoretical support for star-spots migrating to the $\mathrm{L} 1$ region. Coupled with rapidly rotating, single late-type star observations showing intense magnetic cycles, all indications were that CV secondaries should be extremely magnetically active and exhibit "solar cycles".

Recently, low state observations of polars have shown fairly direct evidence for star-spots on their surface (Howell et al. 2000) or TiO absorption attributable to star-spots (Webb et al. 2002). Hessman (this volume) has attempted to detail a possible mechanism which may be responsible for the changing magnetic structures and thus the observed mass transfer variations.

Based on the observational evidence presented in Howell et al. (2000) for the polar ST LMi (while in a low state) a theoretical model has emerged. Noting the lack of ellipsoidal variations from the secondary star during ST LMi's low state, Howell et al. calculated some basic parameters related to the necessary conditions to fulfill mass transfer requirements. Using the basic assumptions outlined in the standard model for CV evolution (see Howell et al. 2001) and relatively simple physical arguments about what is needed to provide the normal mass transfer rate as a function of orbital period, a toy model was produced.

Fig. 1 provides the basic ideas formulated in this model. For long period CVs $\left(\mathrm{P}_{\text {orb }} \geq 3 \mathrm{~h}\right.$ ), the photosphere must be in contact with the Roche Lobe in order to provide the necessary mass flow. If the secondary star, even locally, has a field strength of 170 gauss or more, magnetic lines of force can and will remove material from the binary leading to a stellar wind, in agreement with orbital angular momentum loss due to magnetic braking. This field strength is rather modest and it is probable that essentially all long period CVs meet this criteria. For short period CVs, those with periods below the period gap, the toy model makes very different predictions. First, the star must have have a fairly substantial field strength (> 3000 gauss) to enable magnetic wind momentum loss. Secondly, the stellar photosphere has such a high density, that its contact with the Roche surface is not needed to provide the required mass transfer rate. For these low mass stars $\left(\mathrm{M}_{\text {sec }}<0.25 \mathrm{M}_{\odot}\right)$, a standard chromosphere provides the requisite density to satisfy mass transfer needs. The field strength of 3000 gauss or more may seem unlikely, but for such a rapidly rotating star, extrapolation from single star values appears to easily provide the needed field strength.

For a single late $\mathrm{M}$ star, stellar activity cycles are known to provide chromospheres which can "puff up" by 10 to $30 \%$ of the stellar radius (Guinan 1999). Thus, a secondary star in a short period CV could easily be imagined to fill its Roche Lobe with a chromosphere during a high state (the toy model presented in Howell et al. assumes the chromosphere to fill $10 \%$ of the Roche Lobe volume during a high state) and then to shrink back inside, thus decreasing or stopping 


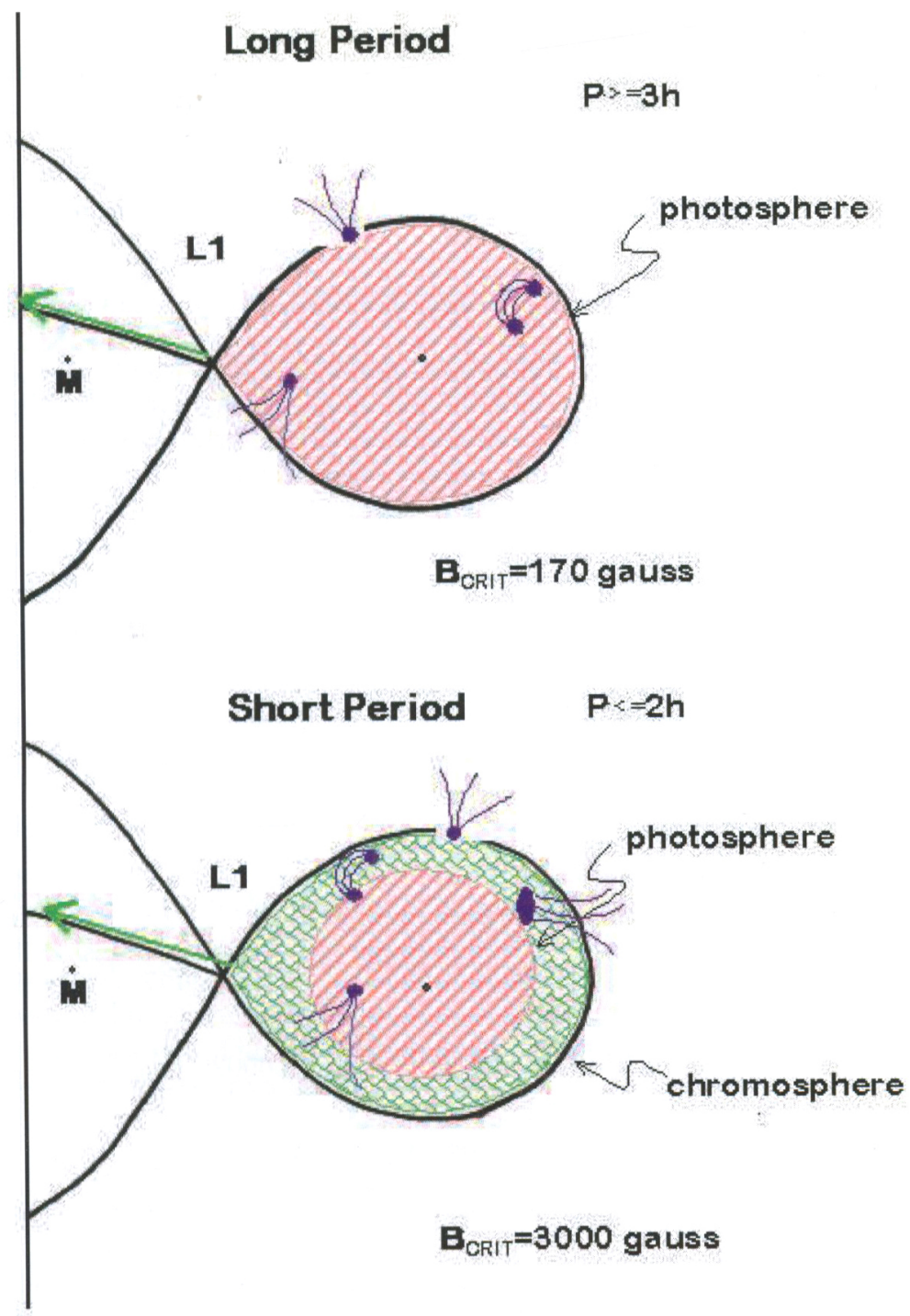

Figure 1. A cartoon view of the toy model proposed by Howell et al., (2000) for general mass transfer in CVs. Long period systems fill the Roche Lobes with their photosphere while short period CVs need only their chromospheres filling the Roche volume. See text for details.

mass transfer during a low state. These modulations would therefore be highly correlated with magnetic activity cycles in the star.

Recent observations of the pre-CV V471 Tau (Rottler et al. 2002) also appear to provide evidence for stellar dynamo modulation of magnetic cycles 
on the secondary star leading to changes in the level of chromospheric activity. While all the above ideas related to some form of stellar activity mechanism similar to solar cycles are appealing, the use of plane-parallel, non-rotating, normal stellar atmosphere models backed by few observations are, at best, only a starting point. Proper interior and atmospheric models of the secondary stars in CVs and additional phase resolved spectroscopy of the secondaries are needed.

\section{Theoretical Assumptions about the Secondary Stars}

Keeping to our belief that the "standard model" for CV evolution is valid, we find that for CVs with periods below the period gap, it is possible for them to be pre- or post-orbital period minimum systems. If pre-orbital period minimum, the secondary stars are essentially normal stars in terms of their mass-radius relation but probably differ from normal main sequence stars in terms of their atmospheric structure, chemical composition, and effective temperature for a given mass. Relations between their mass, radius, and orbital period are presented in Howell et al. (2001).

For post-orbital period minimum CVs, the secondary stars are degenerate. That is, they have masses less than $\sim 0.06 \mathrm{M}_{\odot}$, no core energy generation, and stellar structures of a completely unknown kind. These secondary stars are akin to brown dwarfs in some ways, but one must keep in mind that they formed very differently from field brown dwarfs.

The relations between the mass, radius, and orbital period for post-orbital period minimum CVs are:

$$
\begin{aligned}
& M_{\text {don }}=0.2053 \quad 0.2131 \cdot P_{\text {orb }}+0.08762 \cdot P_{\text {orb }}^{2} \quad 0.01282 \cdot P_{\text {orb }}^{3} \\
& R_{d o n}=0.08195+0.01441 \cdot P_{\text {orb }} \quad 0.0002 \cdot P_{o r b}^{2} \\
& R_{\text {don }}=0.1439 \quad 1.703 \cdot M_{d o n}+16.80 \cdot M_{d o n}^{2}
\end{aligned}
$$

where mass and radius are in solar units and orbital period is in hours (see Howell et al. (2001).

While no observational confirmation of these relationships (as well as the orbital period - effective temperature relationship; see Mennickent \& Diaz 2001) is as yet absolute, we will see below that the secondary star in EF Eri and a few others seem to be strong candidates for post-orbital period minimum CVs.

\section{EF Eri}

EF Eridanus has been in a low state for over seven years now. Optical and IR photometry and spectroscopy obtained over the past year provide an interesting picture for the secondary star. The optical photometry shows a low level modulation ( $0.1 \mathrm{mag})$ as the heated polar region gets self-eclipsed by the white dwarf. Optical spectroscopy (Figure 2) shows nothing more than a cool, magnetic white dwarf with all of the usual signs of mass transfer being absent. 


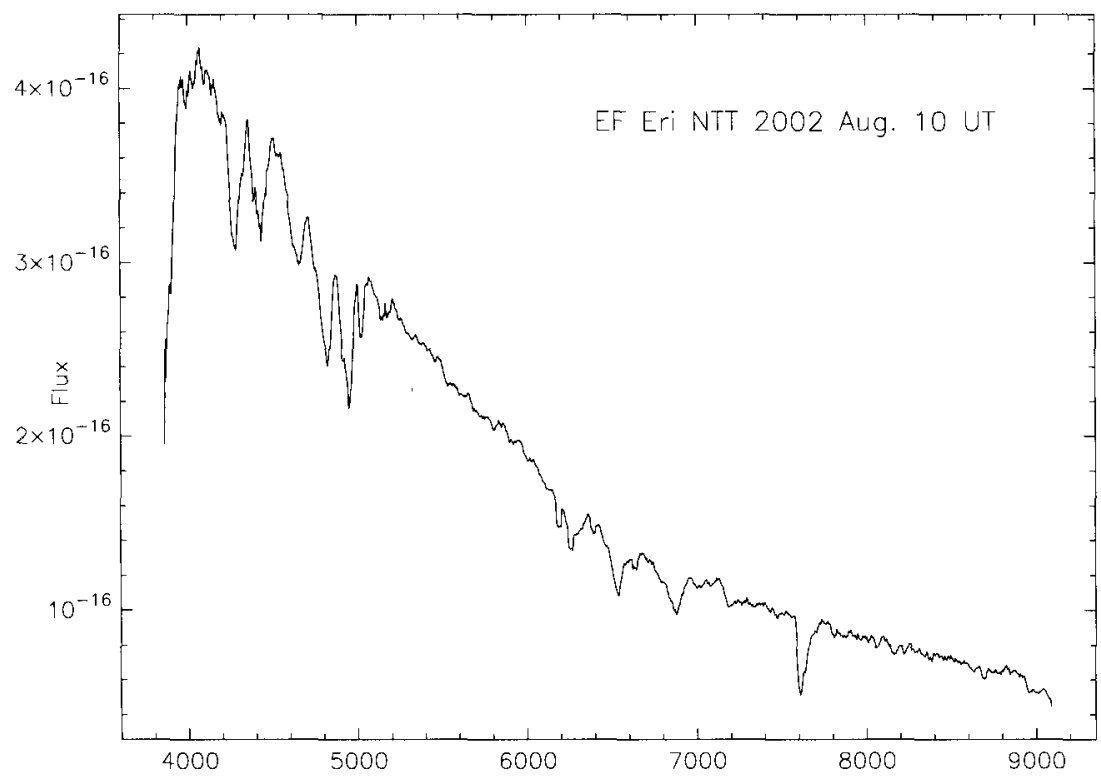

Wovelength (A)

Figure 2. EF Eri observed at the NTT in August 2002. This spectrum, seven years into its current low state, reveals no emission lines or indication of residual cyclotron emission and clearly shows the Zeeman split Balmer lines. Compare this spectrum with one of similar wavelength coverage shown in Beuermann et al., (2000), 3 years into the present low state, which has $\mathrm{H} \alpha$ in emission and cyclotron modulation in the near-IR. The spectral energy distribution in the NTT spectrum is fit fairly well by a $9500 \mathrm{~K}$ white dwarf.

Infrared photometry reveals in $\mathrm{J}$ a similar light curve structure to that seen in the optical. However, $\mathrm{H}$ and $\mathrm{K}$ bands are not only completely different and opposite in phase, but they show a single large modulation of nearly 1 mag. No ellipsoidal variations are observed. IR spectroscopy obtained at UKIRT and recently at Gemini North, show a secondary star which has an apparent temperature on the white dwarf facing hemisphere of $\sim 1800 \mathrm{~K}$ but is only about $900 \mathrm{~K}$ on the opposite side. These temperatures roughly correspond to an M8V star and a L8V brown dwarf. Assuming $\mathrm{M}_{d o n}=0.045 \mathrm{M}_{\odot}$, EF Eri is a cataclysmic variable containing a brown dwarf-like secondary.

Detailed interpretation of these data and model results for EF Eri's component stars are presented in Harrison et al. (2003). Figure 3 illustrates a schematic model of the EF Eri system showing the particulars as well as its size in relation to the planet Jupiter and to the Earth-Moon distance. 


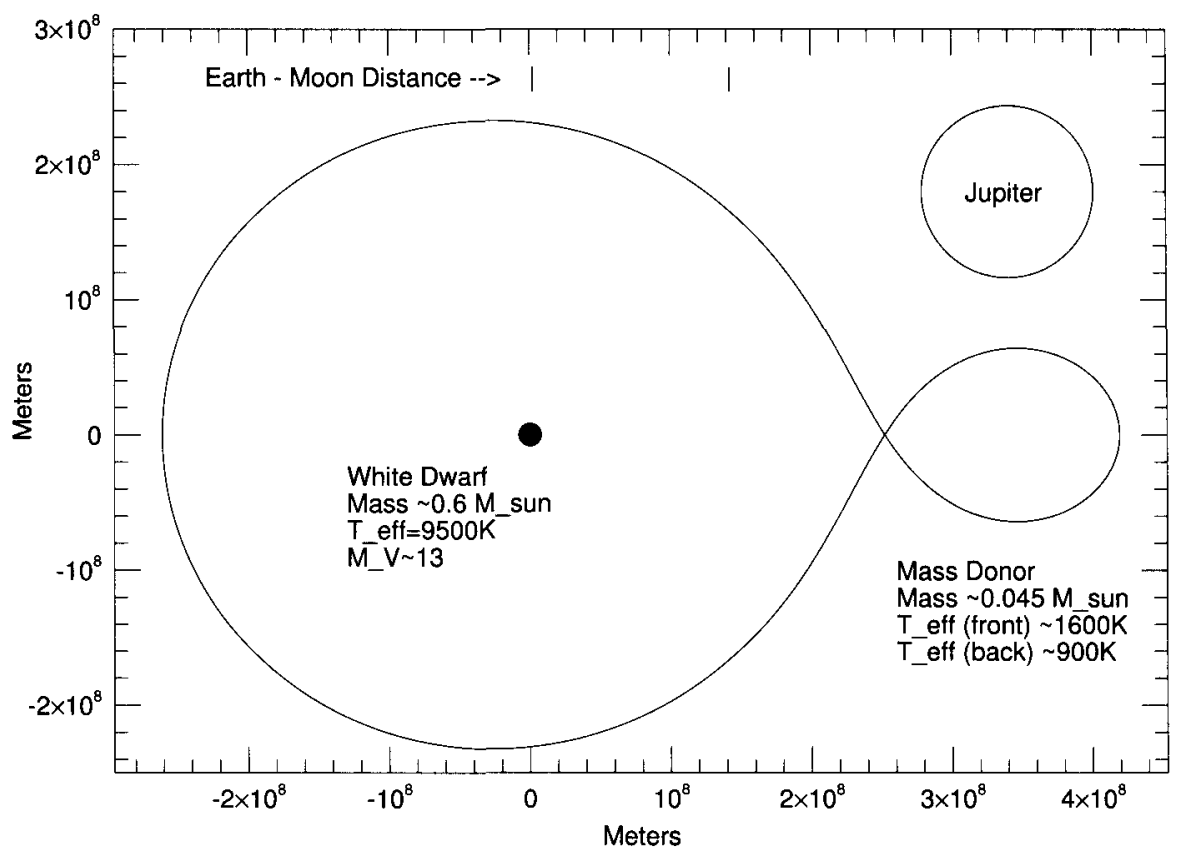

Figure 3. EF Eri shown to scale with the Harrison et al., (2003) parameters listed. Additionally, the size of the planet Jupiter and the Earth-Moon distance are drawn in. Note that even the white dwarf size can be shown to scale in this very compact $81 \mathrm{~min}$ orbital period binary.

\section{Summary}

Low state observations of the polar EF Eri in the optical and particularly in the infrared have revealed that it contains a very odd, low mass secondary star. An object of seemingly unique characteristics with completely unknown interior and atmospheric structure. More observations are needed of EF Eri itself, as well as other candidate CVs which may contain similar odd mass donor stars. Polars, during their low states, provide an ideal opportunity to directly observe the secondary star with little or no interference from accretion flux. This is in contrast to IR spectral observations of even the shortest period, low mass transfer rate disk systems which have, to date, essentially thwarted all efforts to obtain essentially any (good quality) spectra of their secondary star.

The list of good suspects which probably contain brown dwarf-like secondaries includes, in addition to EF Eri, LL And, AL Com, V592 Her, WZ Sge, and 1RXS J1050-1404. All of these systems, except EF Eri, are TOADs (Howell et al. 1995) and therefore the remaining members of this class of CV should be regarded as likely candidates as well.

Acknowledgments. The author wishes to thank Tom Harrison and Elena Mason for observational camaraderie and Kami Leanord, Elaine Owens, and Gil 
Esquerdo for their help with Fig. 1 which once again showed why astronomers "don't do windows".

\section{References}

Beuermann, K., Wheatly, P., Ramsey, G., Euchner, F., \& Gänsicke, B.T. 2000, A\&A, 354, L49

Bianchini, A. 1992, in "Vina del Mar Workshop on Cataclysmic Variable Stars", ed. Nikolaus Vogt, ASP Conf. Series Vol. 29, 284

Guinan, E. 1999, private communication

Harrison, T., Howell, S.B., Huber, M., Osborne, Heather L., Holtzman, J.A., Cash, J.L., \& Gelino, D.M. 2003, AJ, 125, 2609

Hessman F.V., Gänsicke, B.T., \& Mattei, J. A. 2000, A\&A, 361, 952

Howell, S.B., Nelson, L., \& Rappaport, S. 2001, ApJ, 550, 897

Howell, S.B., Ciardi, D., Dhillon, V., \& Skidmore, W. 2000, ApJ, 530, 904

Howell, S.B., Szkody, P., \& Cannizzo, J. 1995, ApJ, 439, 337

King, A. \& Cannizzo, J. 1998, ApJ, 499, 348

Mennickent, R., \& Diaz, M. 2002, MNRAS, 336, 767

Rottler, L., Batalha, C., Young, A., \& Vogt, S. 2002, A\&A, 392, 535

Webb, N., Naylor, T., \& Jeffries, R. 2002, ApJ, 568, L45 